\title{
Fehllage zentraler Venenkatheter in der Arteria subclavia: zuverlässige Entfernung mit einem perkutanen Verschlusssystem (AngioSeal)
}

\author{
Misplacement of Central Venous Catheters in the Subclavian Artery: \\ Safe Retrieval with a Percutaneous Closure Device (AngioSeal)
}

Autoren

Institute
C. Bangard, D-H. Chang ${ }^{1}$, M. Libicher ${ }^{2}$, H. Bovenschulte ${ }^{1}$, M. Kochanek ${ }^{3}$, H. Reuter ${ }^{4}$, D. Maintz ${ }^{1}$

Institut und Poliklinik für Radiologische Diagnostik, Uniklinik Köln

Institut für diagnostische und interventionelle Radiologie, Diakonie-Klinikum Schwäbisch Hall

Medizinische Klinik I für Innere Medizin, Uniklinik Köln

Medizinische Klinik III für Innere Medizin, Uniklinik Köln
Key words

- interventional procedures

- subclavian artery

- vascular closure device

- Angioseal

- obesity

- body mass index eingereicht 13.8 .2012

akzeptiert $\quad 5.1 .2013$

Bibliografie

DOI http://dx.doi.org/

10.1055/s-0032-1330738

Online-Publikation: 28.2.2013

Fortschr Röntgenstr 2013; 185:

546-549 @ Georg Thieme

Verlag KG Stuttgart . New York . ISSN 1438-9029

Korrespondenzadresse

Priv.-Doz. Dr.

Christopher Bangard

Institut und Poliklinik für

Radiologische Diagnostik,

Uniklinik Köln

Kerpener Str. 62

50924 Köln

Tel.: ++ 49/221/4785060

Fax: ++49/221/4786787

christopher.bangard@uk-koeln. de

\section{Zusammenfassung}

$\boldsymbol{\nabla}$

Ziel: Machbarkeitsstudie zur Entfernung fehlliegender zentraler Venenkatheter in der Arteria subclavia mit einem perkutanen Verschlusssystem (AngioSeal).

Material und Methoden: Mittels Datenbankanalyse wurden in einer universitären Einrichtung im Zeitraum Januar 2008 bis Juni 2012 insgesamt 7 Patienten (6 Frauen, 1 Kleinkind) ermittelt, bei denen ein perkutaner Verschluss der arteriellen Punktionsstelle eines versehentlich in die A. subclavia eingebrachten zentralvenösen Katheters $(4 \times 7 \mathrm{~F}, 1 \times 8 \mathrm{~F}, 2 \times 13 \mathrm{~F})$ mittels AngioSeal $8 \mathrm{~F}$ durchgeführt worden war. Entsprechend dem Body-Mass-Index (BMI) waren 3 Patienten adipös (BMI $2 \times 35,7$ und $1 \times 33,0$ ). Bei beiden 13 F-Kathetern wurde ein wie in der Literatur beschriebenes ballonunterstütztes Verfahren verwendet. Im Falle eines 21 Monate alten Kleinkindes kam mit Freilegung des Broviakkatheters eine assistiert operative perkutane Katheterentfernung zum Einsatz. Bei 5 der 7 Patienten erfolgte eine Erfolgskontrolle durch transfemorale i.a. DSA, bei 2 Patienten klinisch.

Ergebnisse: In allen Fällen wurde der fehlliegende Katheter erfolgreich entfernt. In allen 7 Fällen kam es zu einer sofortigen Bluttrockenheit. Komplikationen traten nicht auf.

Schlussfolgerung: Die Entfernung fehlliegender zentraler Venenkatheter in der Arteria subclavia mit einem perkutanen Verschlusssystem (Angio Seal) ist sicher. Auch bei adipösen Patienten kann die perkutane Katheterentfernung als Verfahren der ersten Wahl eingesetzt werden.

\section{Einleitung}

Die Anlage eines zentralvenösen Katheters ist ein medizinischer Routineeingriff. Bei Intensivpatienten ohne Notfallindikation werden dennoch

\section{Abstract \\ $\nabla$}

Purpose: To evaluate the feasibility of retrieval of misplaced central venous catheters in the subclavian artery with a percutaneous closure device (AngioSeal).

Materials and Methods: According to the radiologic information system of a university hospital, 7 patients ( 6 women, 1 child) underwent removal of a misplaced central venous catheter $(4 \times 7 \mathrm{~F}$, $1 \times 8 \mathrm{~F}, 2 \times 13 \mathrm{~F})$ in the subclavian artery between January 2008 and June 2012 with the $8 \mathrm{~F}$ percutaneous closure device AngioSeal. The body mass index (BMI) of 7 patients was available, 3 of whom were obese (BMI $2 \times 35.7$ and $1 \times 33.0$ ). Both $13 \mathrm{~F}$ catheters were removed in a balloonsupported manner as described elsewhere. The Broviak catheter of a 21-month-old boy was removed by surgical support with preparation of the catheter to its vessel entry. Success control was done by a transfemoral DSA in 5 of the 7 patients. 2 patients received clinical follow-up.

Results: In all cases the misplaced catheter could be removed successfully. In all 7 patients, there was no arterial bleeding after catheter removal. Complications were not observed.

Conclusion: Removal of misplaced central venous catheters in the subclavian artery by the percutaneous closure device AngioSeal is safe. Percutaneous catheter removal should be regarded as the method of choice, even in obese patients.
Komplikationsraten von über $5 \%$ berichtet. Neben dem Pneumothorax ist die arterielle Fehlpunktion eine der häufigsten Komplikationen. Die Rate arterieller Fehlpunktionen der A. subclavia ohne Ultraschallkontrolle liegt bei 5,4\% [1]. Auch 
Tab. 1 Patienten, Kathetergrößen, Katheterwinkel und Interventionsverlauf.

\begin{tabular}{|c|c|c|c|c|c|c|c|c|c|c|c|c|}
\hline Patient & Geschlecht & $\begin{array}{l}\text { Alter } \\
\text { (J) }\end{array}$ & $\begin{array}{l}\text { Gewicht } \\
\text { (kg) }\end{array}$ & $\begin{array}{l}\text { Größe } \\
\text { (m) }\end{array}$ & $\begin{array}{l}\text { BMI } \\
\left(\mathrm{kg} / \mathrm{m}^{2}\right)\end{array}$ & $\begin{array}{l}\text { Katheter } \\
\text { (F) }\end{array}$ & $\begin{array}{l}\text { Winkel } \\
\left({ }^{\circ}\right)\end{array}$ & $\begin{array}{l}\text { Liegedauer } \\
\text { (Stunden) }\end{array}$ & Kontrolle & Erfolg & $\begin{array}{l}\text { Kompli- } \\
\text { kationen }\end{array}$ & Besonderheiten \\
\hline 1 & w & 87 & 50,0 & 1,65 & 18,4 & 7 & 166,3 & 13,5 & i. a. DSA & ja & keine & keine \\
\hline 2 & w & 67 & 63,0 & 1,64 & 23,4 & 13 & 159,5 & 23,5 & i. a. DSA & ja & keine & ballonunterstützt \\
\hline 3 & w & 67 & 50,0 & 1,63 & 18,8 & 7 & n. m. & 14 & klinisch & ja & keine & $\begin{array}{l}\text { Intervention am } \\
\text { Bett }\end{array}$ \\
\hline 4 & $\mathrm{~m}$ & 1,75 & 10,0 & 0,84 & 14,2 & 7 & n. m. & 410 & i. a. DSA & ja & keine & assist. operativ \\
\hline 5 & w & 43 & 102,0 & 1,69 & 35,7 & 13 & 126,2 & 20 & i. a. DSA & ja & keine & ballonunterstützt \\
\hline 6 & w & 45 & 79,2 & 1,55 & 33,0 & 8 & 136,2 & 8 & klinisch & ja & keine & keine \\
\hline 7 & w & 60 & 91,5 & 1,66 & 35,7 & 7 & 134,5 & 53 & i. a. DSA & ja & keine & keine \\
\hline
\end{tabular}

w - weiblich, m - männlich, J - Jahre, kg - Kilogramm, m - Meter, BMI - Body-Mass-Index, F - French, ${ }^{\circ}$ - Grad, n. m. - nicht messbar, i. a. DSA - intraarterielle digitale Subtraktionsangiografie, Winkel - Eintrittswinkel des Katheters zum Verlauf der A. subclavia, Liegedauer - Katheterliegedauer vor Extraktion.

eine ultraschallgesteuerte Katheteranlage kann eine arterielle Fehlpunktion nicht immer sicher verhindern, da je nach Füllungszustand der Vene und Rigidität der Venenwand ein notwendiger schneller Nadelvorschub zu einer Venenperforation mit arterieller Fehlpunktion führen kann. Normalerweise wird die arterielle Fehlpunktion bemerkt und durch Zurückziehen der Punktionsnadel mit nachfolgender Kompression die Punktionsstelle verschlossen. Wird die Fehlpunktion jedoch nicht bemerkt und ein Katheter eingelegt, kann das Entfernen des Katheters aufgrund der schlechten Komprimierbarkeit der A. subclavia durch Lage unter dem Schlüsselbein zu einer lebensbedrohlichen Blutung führen [2, 3]. Die operative Katheterentfernung ist bei instabilen Patienten nicht immer möglich und auch bei kreislaufstabilen Patienten durch die oft notwenige partielle Resektion des Schlüsselbeins oder einer Rippe ein komplikationsträchtiger Eingriff [4].

Seit mehr als einer Dekade kommen perkutane arterielle Verschlusssysteme zum Einsatz. Diese sind für die Anwendung nach retrograder Punktion der A. femoralis communis zugelassen [5]. Es sind mehrere Systeme mit unterschiedlichen Verschlussmechanismen erhältlich: Nitinolclip-basiert (z. B. StarClose - Abbott Laboratories, Illionois, USA), Naht-basiert (z. B. PerClose, Abbott Laboratories) und Kollagen-basiert (z. B. AngioSeal, St. Jude medical, Minnetonka, USA). Aufgrund der hohen Effektivität und geringen Komplikationsrate der perkutanen Verschlusssysteme findet seit Langem auch ein „off label use“ bei dem Verschluss antegrader Punktionen der A. femoralis communis statt. Die primär erfolgreiche Blutstillung retrograder Punktionen der A. femoralis communis ist mit 97,3\% sehr hoch, wobei von den $2,7 \%$ Versagern ca. 7\% Komplikationen wie größere Leistenhämatome, AVFisteln, Pseudoaneurysmen, Beinischämien oder retroperitoneale Hämatome entwickeln [6]. Einige Studien konnten die Wirksamkeit der Verschlusssysteme nach antegrader femoraler Punktion zeigen [7-9]. In einer prospektiven Studie von Minko und Kollegen war Adipositas eine unabhängige Variable für einen Misserfolg bei dem Verschluss antegrader Punktionen der A. femoralis communis [9]. Der perkutane Verschluss nach Fehlpunktion der A. carotis interna [10] oder der A. subclavia ist in wenigen Fallberichten mit max. 4 oder 5 Patienten beschrieben [11 18]. Angaben zum Body-Mass-Index (BMI) der Patienten existieren meist nicht.

Ziel der vorliegenden Arbeit war daher eine systematische Datenbankanalyse einer universitären Einrichtung mit Bestimmung der Erfolgsrate der Entfernung fehlliegender zentraler Venenkatheter in der Arteria subclavia mit einem perkutanen Verschluss- system (AngioSeal) unter Berücksichtigung des BMI der Patienten.

\section{Material und Methoden \\ $\nabla$}

Das radiologische Informationssystem wurde für den Zeitraum Januar 2008 bis Juni 2012 einer Datenbankanalyse unterzogen. Als Suchbegriffe wurden die Wörter „Angioseal“ und „A. subclavia“ verwendet. Bei 7 Patienten (6 Frauen, 1 Kleinkind) erfolgte in diesem Zeitraum ein perkutaner Verschluss der arteriellen Punktionsstelle eines versehentlich in die A. subclavia eingebrachten zentralvenösen Katheters mittels AngioSeal ${ }^{\mathrm{TM}}$ STS Plus ${ }^{\mathrm{TM}}$ (St. Jude Medical, St. Paul, Minnetonka, USA). Von allen 7 Patienten waren Gewicht und Körpergröße bekannt, sodass der BMI (kg/ $\mathrm{m}^{2}$ ) berechnet werden konnte. Die Dimensionen der fehlliegenden Katheter variierten zwischen 7-13 F ( $\bullet$ Tab. 1).

AngioSeal ist ein kollagenbasiertes perkutanes Verschlusssystem, das für den Verschluss retrograder arterieller Punktionen der A. femoralis communis zugelassen ist. 6F- und 8F-Bestecke stehen zur Verfügung. Ein aus bioresorbierbarem Polymer bestehender Gefäßanker richtet sich als Widerlager im Gefäß auf und ist mit einem resorbierbaren Kollagenpfropf verbunden. Durch Zug am intraluminalen Gefäßanker und einem gleichzeitigen Druck von außen auf den Kollagenpfropf wird die arterielle Punktionsstelle mechanisch verschlossen. Der intraluminale Gefäßanker und der extraluminale Kollagenpfropf fassen die Gefäßwand in ihrer Mitte sandwichartig ein. Die Einführschleuse des AngioSeal-Bestecks besitzt am distalen Ende eine kleine Eintrittsöffnung, über die bei Eintritt in das Gefäßlumen durch einen Blutaustritt am proximalen Ende der Schleuse die korrekte intraluminale Position an der Gefäßwand angezeigt wird.

Die Entfernung der fehlliegenden Katheter wurde standardisiert unter Einhaltung der Herstellerangaben durchgeführt. Ein steifer 0,035-inch-Führungsdraht (Fixed Core Wire Guide, Safe-T J-curved, $160 \mathrm{~cm}$, Fa. Cook Inc., Bloomington, USA) wurde zum Wechsel des Katheters gegen die Einführschleuse des AngioSeal-Bestecks verwendet. Alternativ wurde ein steifer hydrophil beschichteter 0,035-inch-Führungsdraht (Radiofocus ${ }^{\circledR}$ Guide Wire, stiff type angled, $260 \mathrm{~cm}$, Terumo Corp., Tokyo, Japan) eingesetzt. In einem Fall eines erst 21 Monate alten Kleinkinds erfolgte zur besseren Kontrolle eine operative Freilegung der Eintrittsstelle des Katheters und somit ein assistiert operativer perkutaner Verschluss. Eine chirurgische Gefäßnaht erfolgte jedoch nicht. Bei 2 Patienten wurde zur Fixierung des Gefäßankers und zur Reduktion des Blutflusses bei der Entfernung der 13F-Shaldonkatheter durch das unterdimen- 
sionierte 8F-Angioseal-Besteck ein Ballonkatheter (Opta pro, $10 \times 40 \mathrm{~mm}$ bzw. $8 \times 40 \mathrm{~mm}$, Fa. Cordis/Johnson \& Johnson, USA) über die Eintrittsstelle des Katheters in die A. subclavia distal des Abgangs der A. vertebralis platziert und für 2 min partiell inflatiert $[17,18]$. Es erfolgte keine Heparinisierung während der Angiografien. Der technische Erfolg wurde in 5 der 7 Patienten periinterventionell durch eine transfemorale i.a. DSA kontrolliert. Die transfemorale Punktion diente auch zur Absicherung, falls bei Misserfolg der Intervention und akuter Blutung ein Okklusionsballon oder die Freisetzung einer Gefäßprothese notwendig geworden wäre. In 2 Fällen erfolgte die Erfolgskontrolle klinisch durch Inspektion und Palpation der Punktionsstelle sowie regelmäßige Hb-Kontrollen.

Bei den 6 Patienten, die eine intraarterielle Angiografie erhielten, sollte der Winkel digital im PACS gemessen werden, den der fehlliegende Katheter in seinem subkutanen Verlauf mit dem intraarteriellen Verlauf bildete, um den Punktionswinkel zur A. subclavia abzuschätzen. Das Zeitintervall von der arteriellen Fehlpunktion bis zum interventionellen Verschluss der Punktionsstelle wurde erfasst.

\section{Ergebnisse}

$\nabla$

In allen Fällen wurde das 8F-Angioseal-Besteck erfolgreich verwendet ( $\bullet$ Tab. 1 ). In 6 Fällen konnte die AngioSeal-Schleuse über den 0,035 inch Fixed Core Wire eingewechselt werden. In einem Fall war das Lumen des Katheters auch nach mehrfachem Spülen nicht mehr gleitfähig genug. Hier musste der hydrophile 0,035 inch Radiofocus Guide Wire verwendet werden. Im Falle des 21 Monate alten Kleinkinds lag bereits vor Entfernung des 7F-Broviakkatheters ein kollateralisierter Abgangsverschluss der linken A. subclavia vor, der auch nach erfolgreichem Verschluss der Punktionsstelle fortbestand und mit dem Wechseldraht für die AngioSeal-Schleuse problemlos passiert werden konnte. Der Verschluss der A. subclavia resultierte vermutlich aus einer primär okklusiven Katheterlage. In allen 7 Fällen kam es zu einer sofortigen Bluttrockenheit. Komplikationen traten nicht auf (๑ Abb. 1). Die 2 ausschließlich klinisch nachkontrollierten Patienten zeigten stabile Hämoglobinwerte und unauffällige Palpations- und Inspektionsbefunde der Punktionsstelle. Einer dieser beiden Patienten war eine nicht transportfähige Frau, die wegen einer ausgeprägten Herzinsuffizienz eine extrakorporale Membranoxygenierung (ECMO) erhalten sollte. Daher erfolgte der perkutane Verschluss auf Intensivstation ohne begleitende bildgebende Kontrolle. Bei dem Kleinkind konnte aufgrund des sehr kurzstreckigen subkutanen Verlaufs des Broviakkatheters der Punktionswinkel zur A. subclavia nachträglich nicht mehr bestimmt werden. Der Winkel der fehlliegenden Katheter der 5 auswertbaren Patienten lag zwischen $120^{\circ}$ und $180^{\circ}(144,5$ $\pm 17,3$ Grad) ( $\triangle$ Abb.2). Die Liegedauer des Katheters von der Fehlpunktion bis zur Extraktion betrug im Mittel 77,4h (min. 8 h, max. 410 h) (॰ Tab. 1).

\section{Diskussion \\ $\nabla$}

Die arterielle Katheterfehllage bei Anlage eines zentralvenösen Katheters ist eine seltene Komplikation. In unserer universitären Einrichtung kam es in 4,5 Jahren zu 7 arteriellen Katheterfehllagen. Andere berichten über einen einzigen Fall innerhalb von 20 Jahren [19]. Hierbei liegen oft besondere Gegebenheiten vor,
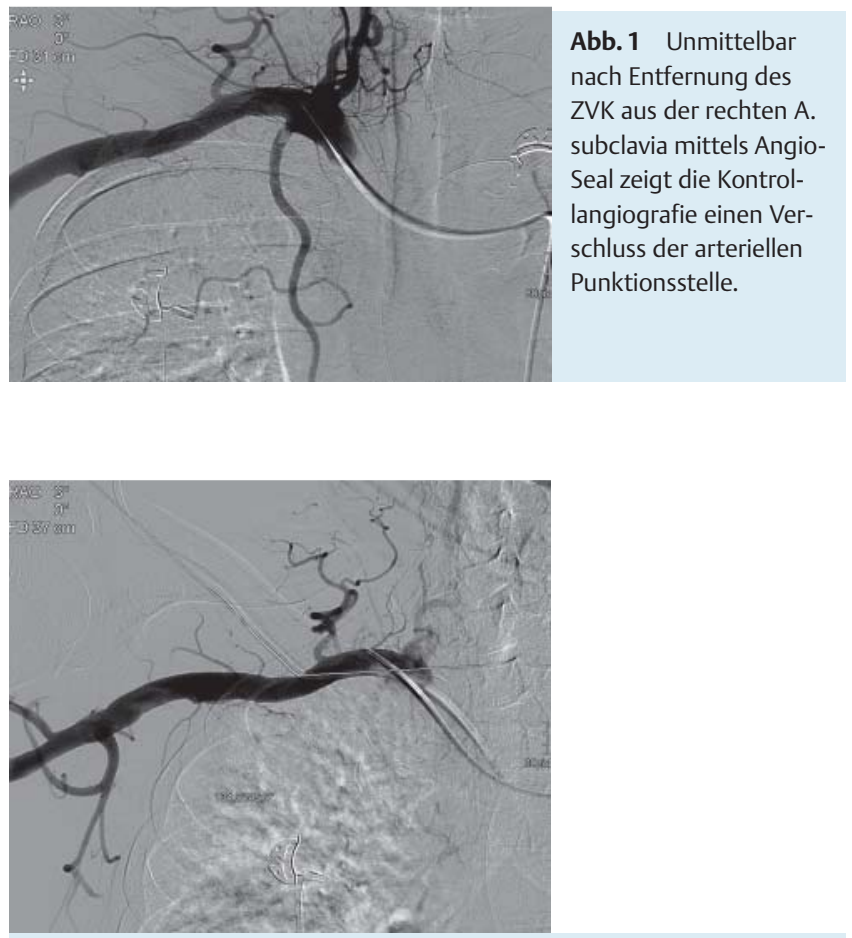

Abb. 2 Der fehlliegende ZVK in der rechten A. subclavia bildet in seinem subkutanen Verlauf einen Winkel von $134,5^{\circ}$ mit dem intraarteriellen Verlauf. Dieser flache Winkel gewährleistet ein problemloses Einführen der AngioSeal-Schleuse ohne Abknicken, sodass der Kollagenpropf ungehindert vorgeschoben werden kann.

wie die Anlage während kardiopulmonaler Reanimation oder unter erschwerten Bedingungen durch den Notarzt am Unfallort ( 2 unserer berichteten Fälle). Drei unserer Patienten waren adipös. Adipositas ist eine unabhängige Variable für ein gehäuftes Auftreten von Komplikationen, beispielsweise nach abdominellen Operationen oder gefäßchirurgischen Eingriffen [20, 21], und erschwert die Anlage zentralvenöser Katheter. Adipositas ist ein unabhängiger Risikofaktor für eine insuffiziente Blutstillung bei perkutanem Verschluss antegrader Punktionen der A. femoralis communis mittels AngioSeal. Alle 9 Patienten, bei denen in der prospektiven Studie von Minko und Kollegen die weiche Schleuse des AngioSeal-Bestecks abknickte und dadurch ein Vorschieben des Kollagenpfropfs nicht möglich war, waren adipös und hatten einen mittleren BMI von 31,7 [9]. Hier spielt der steilere Punktionswinkel bei Adipösen bei antegrader Punktion der A. femoralis communis eine entscheidende Rolle. Der Punktionswinkel bei Anlage eines zentralvenösen Katheters über die V. subclavia ist im Gegensatz dazu nicht zwangsläufig steiler, wenn ein Patient adipös ist. Das könnte erklären, warum in der vorliegenden Arbeit trotz eines mittleren BMI von 34,8 aller 3 adipösen Patienten der perkutane Verschluss mittels AngioSeal erfolgreich war. Sowohl die androide Fettverteilung bei Adipositas bei Männern (abdominell betont) als auch die gynoide Fettverteilung bei Frauen (femorogluteal betont) haben verhältnismäßig mehr Einfluss auf die inguinale Punktionsstelle als auf die subklavikuläre Punktionsstelle [22], weshalb ein erhöhter BMI kein Risikofaktor beim perkutanen Verschluss subklavikulärer Punktionsstellen zu sein scheint. Ein weiterer Grund könnte in der vorteilhafteren Anatomie der Schlüsselbeinregion verglichen mit der Leistenregion bezüglich des Gefäßverlaufs und damit der Winkelung der AngioSeal-Schleuse liegen. Insbesondere bei Adi- 
pösen kommt es nicht selten zu einem Winkel des Schleusenverlaufs nach antegrader Punktion der A. femoralis communis von kleiner als $120^{\circ}$, da die Verlängerung des Punktionswinkels nicht die A. femoralis superficialis, sondern die A. profunda femoris ist. Der von lateral nach medial kraniokaudale Verlauf der A. subclavia hingegen stellt einen vorteilhaften Schleusenwinkel von mehr als $120^{\circ}$ sicher, was in 5 Fällen gezeigt werden konnte. Die Schleuse knickte daher nicht ab und der Kollagenpfropf konnte ungehindert vorgeschoben werden. Trotz der100\%igen Erfolgsrate bei den beschriebenen 7 Fällen ist darauf zu achten, in kritischen Situationen bei Adipositas oder gefährlichen Lokalisationen wie in der A. subclavia nicht den kurzen Draht des AngioSeal-Bestecks zu verwenden, sondern einen langen steifen Wechseldraht. Oft muss Zug auf den Draht ausgeübt werden, um die weiche AngioSeal-Schleuse nach intravasal vorschieben zu können, sodass der Draht zurückgezogen wird. Im Falle eines zu kurzen Drahtes kann das zum Verlust der intravasalen Drahtlage führen. Ein langer Draht ermöglicht, über einen Wechselkatheter einen noch steiferen Draht einzuwechseln, wenn ein Vorschieben der AngioSeal-Schleuse über den eingebrachten Draht nicht gelingt. Die ballonassistierte Katheterentfernung ist laut Literatur bei unterdimensionierten perkutanen Verschlusssystemen $a b$ einer Kathetergröße von größer $10 \mathrm{~F}$ zu empfehlen. Eine Heparinisierung während der 1- bis 2-minütigen Balloninflation erfolgte nicht $[17,18]$.

Tran und Kollegen konnten mit dem clipbasierten perkutanen Verschlusssystem Starclose in einer der größten Fallserien mit 5 Patienten ebenfalls eine 100\%ige Erfolgsrate berichten [14]. Nach den ersten positiven Erfahrungen wurden einige der Katheterentfernungen auch am Patientenbett durchgeführt. Dennoch wird empfohlen, diese Technik nicht routinemäßig am Patientenbett durchzuführen, da femoral auch einige fehlgeschlagene Verschlussversuche auftraten. Die Durchführung in der Angiografieeinheit mit optimaler Kontrolle unter Durchleuchtung ist zu bevorzugen. Eine exakte Darstellung der Lagebeziehung des Kathetereintrittspunkts zu angrenzenden sensiblen Gefäßen wie die A. vertebralis ist so gewährleistet [12]. Sollte wie in einem unserer Fälle keine Transportfähigkeit des Patienten bestehen, so kann der perkutane Verschluss am Patientenbett erfolgen. Vor Beginn der Intervention am Bett kann eine Röntgenkassette unter dem Thorax des Patienten platziert werden, die notfalls helfen kann, durch eine Thoraxaufnahme periinterventionell auftretende Probleme aufzudecken.

Limitationen der vorliegenden Arbeit sind die geringe Patientenzahl, die Heterogenität des Durchmessers der entfernten Katheter, die Heterogenität des Alters der Patienten und der begleitend zur Verschlusssystemfreisetzung durchgeführten Maßnahmen.

Zusammenfassend lässt sich feststellen, dass die seltene arterielle Fehllage eines Katheters in der A. subclavia durch Verwendung des perkutanen Verschlusssystems AngioSeal sicher behoben werden kann. Ein erhöhter BMI scheint kein Risikofaktor beim perkutanen Verschluss subklavikulärer Punktionsstellen zu sein.

\section{Literatur}

1 Fragou M, Gravvanis A, Dimitriou V et al. Real-time ultrasound-guided subclavian vein cannulation versus the landmark method in critical care patients: a prospective randomized study. Crit Care Med 2011; 39: $1607-1612$

2 Guilbert MC, Elkouri S, Bracco D et al. Arterial trauma during central venous catheter insertion: Case series, review and proposed algorithm. J Vasc Surg 2008; 48: 918-925

3 Bagwell CE, Salzberg AM, Sonnino RE et al. Potentially lethal complications of central venous catheter placement. J Pediatr Surg 2000; 35: $709-713$

4 Valentine RJ, Wind GG. Anatomic exposures in vascular surgery. Philadelphia: Lippincott, Williams \& Wilkins; 2003, 79-112

5 Kamusella P, Wissgott C, Andresen R. Einsatz eines perkutanen Nahtverschlusssystems nach 6 - $8 \mathrm{~F}$ transfemoralen Zugängen: Ergebnisse von 2200 Patienten. Fortschr Röntgenstr 2012; 184: 311 - 315

6 Bangalore S, Arora N, Resnic FS. Vascular closure device failure: frequency and implications: a propensity-matched analysis. Circ Cardiovasc Interv 2009; 2: 549-556

7 Lupattelli T, Tannouri F, Garaci FG et al. Efficacy and safety of antegrade common femoral artery access closure using the Angio-Seal device: experience with 1889 interventions for critical limb ischemia in diabetic patients. J Endovasc Ther 2010; 17: 366-375

8 Das R, Ahmed K, Athanasiou T et al. Arterial closure devices versus manual compression for femoral haemostasis in interventional radiological procedures: a systematic review and meta-analysis. Cardiovasc Intervent Radiol 2011; 34: 723-738

9 Minko P, Katoh M, Gräber S et al. Obesity: an independent risk factor for insufficient hemostasis using the AngioSeal vascular closure device after antegrade puncture. Cardiovasc Intervent Radiol 2012; 35: 775 778

10 Massière B, von Ristow A, Cury JM et al. Closure of carotid artery puncture site with a percutaneous device. Ann Vasc Surg 2009; 23: 256.e5-256.e7

11 Schütz N, Doll S, Bonvini RF. Erroneous placement of central venous catheter in the subclavian artery: retrieval and successful hemostasis with a femoral closure device. Catheter Cardiovasc Interv 2011; 77: $154-157$

12 Jahromi BS, Tummala RP, Levy EI. Inadvertent subclavian artery catheter placement complicated by stroke: endovascular management and review. Catheter Cardiovasc Interv 2009; 73: 706 - 711

13 Shetty SV, Kwolek CJ, Garasic JM. Percutaneous closure after inadvertent subclavian artery cannulation. Catheter Cardiovasc Interv 2007; 69: $1050-1052$

14 Tran V, Shiferson A, Hingorani AP et al. Use of the StarClose device for closure of inadvertent subclavian artery punctures. Ann Vasc Surg 2009; 23: 688.e11-688.e13

15 Nicholson T, Ettles D, Robinson G. Managing inadvertent arterial catheterization during central venous access procedures. Cardiovasc Intervent Radiol 2004; 27: 21 - 25

16 Chemelli AP, Wiedermann F, Klocker J et al. Endovascular management of inadvertent subclavian artery catheterization during subclavian vein cannulation. J Vasc Interv Radiol 2010; 21: 470-476

17 Bovenschulte H, Chang DH, Michels G et al. Technical note: misplaced $13 \mathrm{~F}$-dialysis catheter in the subclavian artery - controlled removal with an undersized 8F-collagen closure system (AngioSeal ${ }^{\circledR}$ ) and endovascular balloon fixation. Fortschr Röntgenstr 2011; 183: 758 - 760

18 Wildberger JE, Katoh M, Fussen $R$ et al. Technische Mitteilung: Entfernung eines fehl platzierten $12 \mathrm{~F}$ Shaldon Katheters aus der A. subclavia und Abdichtung mittels Verschlusssystem bei temporärer Ballonblockade. Fortschr Röntgenstr 2006; 178: 605-609

19 Micha JP, Goldstein BH, Lindsay SF et al. Subclavian artery puncture repair with Angio-Seal deployment. Gynecol Oncol 2007; 104: 761 - 763

20 Causey MW, Johnson EK, Miller S et al. The impact of obesity on outcomes following major surgery for Crohn's disease: an American College of Surgeons National Surgical Quality Improvement Program assessment. Dis Colon Rectum 2011; 54: 1488-1495

21 Khandanpour N, Armon MP, Foxall $R$ et al. The effects of increasing obesity on outcomes of vascular surgery. Ann Vasc Surg 2009; 23: $310-$ 316

22 Després JP, Lemieux I, Prud 'homme D. Treatment of obesity: need to focus on high risk abdominally obese patients. BMJ 2001; 322: 716-720 